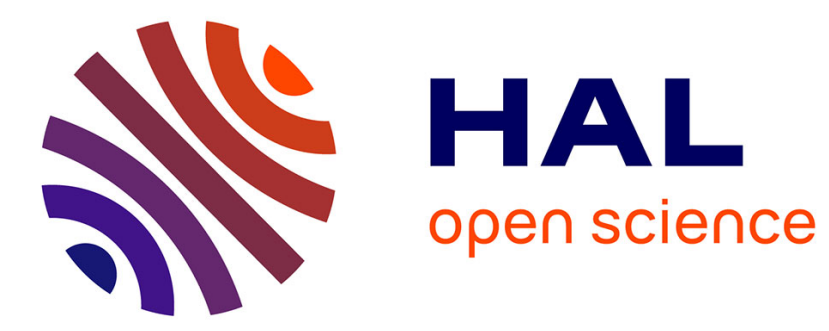

\title{
On the placement of an obstacle so as to optimize the Dirichlet heat trace
}

\author{
Ahmad El Soufi, Evans Harrell
}

\section{To cite this version:}

Ahmad El Soufi, Evans Harrell. On the placement of an obstacle so as to optimize the Dirichlet heat trace. 2014. hal-00947547v1

\section{HAL Id: hal-00947547 \\ https://hal.science/hal-00947547v1}

Preprint submitted on 17 Feb 2014 (v1), last revised 11 Jan 2016 (v2)

HAL is a multi-disciplinary open access archive for the deposit and dissemination of scientific research documents, whether they are published or not. The documents may come from teaching and research institutions in France or abroad, or from public or private research centers.
L'archive ouverte pluridisciplinaire HAL, est destinée au dépôt et à la diffusion de documents scientifiques de niveau recherche, publiés ou non, émanant des établissements d'enseignement et de recherche français ou étrangers, des laboratoires publics ou privés. 


\title{
ON THE PLACEMENT OF AN OBSTACLE SO AS TO OPTIMIZE THE DIRICHLET HEAT TRACE
}

\author{
Ahmad El Soufi \\ Laboratoire de Mathématiques et Physique Théorique, UMR CNRS 7350 \\ Université François Rabelais de Tours, Parc de Grandmont, F-37200 Tours, France \\ EVANS M. HARRELl II \\ School of Mathematics \\ Georgia Institute of Technology, Atlanta GA 30332-0160, USA
}

\begin{abstract}
We prove that among all doubly connected domains of $\mathbb{R}^{n}$ bounded by two spheres of given radii, $Z(t)$, the trace of the heat kernel with Dirichlet boundary conditions, achieves its maximum when the spheres are concentric (i.e., for the spherical shell). The infimum is attained in the limiting situation where the interior sphere is in contact with the outer sphere.

This is shown to be a special case of a more general theorem characterizing the optimal placement of a spherical obstacle inside a domain so as to maximize or minimize the trace of the Dirichlet heat kernel. In this case, for each $t$ the maximizing position of the center of the obstacle belongs to the "heart" of the domain, while the minimizing situation occurs either in the interior of the heart or at a point where the obstacle is in contact with the outer boundary.

Similar statements hold for the optimal positions of the obstacle for any spectral property that can be obtained as a positivitypreserving or positivity-reversing transform of $Z(t)$, as well as for the spectral zeta function and the regularized determinant.
\end{abstract}

\section{INTRODUCTION AND STATEMENT OF RESUltS}

Let $\Omega \subset \mathbb{R}^{n}$ be a bounded $C^{2}$ Euclidean domain and let

$$
\lambda_{1}(\Omega)<\lambda_{2}(\Omega) \leq \lambda_{3}(\Omega) \leq \cdots \leq \lambda_{i}(\Omega) \leq \cdots \rightarrow \infty,
$$

be the sequence of eigenvalues of the Dirichlet realization of the Laplacian $-\Delta$ in $\Omega$, where each eigenvalue is repeated according to its multiplicity. The corresponding "heat operator" $e^{t \Delta}$ has finite trace for all

Date: February 17, 2014.

2000 Mathematics Subject Classification. 35P15, 49R50, 58J50.

Key words and phrases. Dirichlet Laplacian, eigenvalues, heat trace, determinant, obstacle, spherical shell. 
$t>0$ (known in physical literature as the "partition function"), which we denote

$$
Z_{\Omega}(t)=\sum_{k \geq 1} e^{-\lambda_{k}(\Omega) t}
$$

Following Ray and Singer [23] we denote by $\operatorname{det}(\Omega)$ the regularized determinant of the Dirichlet Laplacian in $\Omega$ defined by

$$
\operatorname{det}(\Omega)=\exp \left(-\zeta^{\prime}(0)\right)
$$

where $\zeta$ is the meromorphic extension to the entire complex plane of the spectral zeta function $\sum_{k=1}^{\infty} \lambda_{k}(\Omega)^{-s}$, which is known to be convergent and holomorphic on $\left\{\operatorname{Re} s>\frac{n}{2}\right\}$.

Eigenvalue optimization problems date from Lord Rayleigh's "Theory of Sound" (1894) where it was suggested that the disk should minimize the first eigenvalue $\lambda_{1}$ among all the domains of given measure. Rayleigh's conjecture was proved in any dimension independently by Faber [8] and Krahn [19].

Later, Luttinger [21] proved an isoperimetric result analogous to Faber-Krahn for $Z(t)$, considered as a functional upon the set of bounded Euclidean domains, that is, for any bounded domain $\Omega \subset \mathbb{R}^{n}$ and any $t>0$,

$$
Z_{\Omega}(t) \leq Z_{\Omega^{*}}(t)
$$

where $\Omega^{*}$ is a Euclidean ball whose volume is equal to that of $\Omega$. A similar property was proved in [22] for the regularized determinant of the Laplacian in two dimensions (see $[20,24]$ for other examples of results in this direction).

The case of multi-connected planar domains, i.e. whose boundary admits more than one component, was first considered by Hersch. Using the method of interior parallels, in [16] Hersch proved the following extremal property of annular membranes:

"A doubly connected fixed membrane, bounded by two circles of given radii, has maximum $\lambda_{1}$ when the circles are concentric".

Hersch's result has been extended to a wider class of domains in any dimension by Harrell, Kröger and Kurata [13] and Kesavan [18], whereby the authors consider a fixed domain $D$ from which an "obstacle" of fixed shape, usually spherical, has been excised. The position of the obstacle is allowed to vary, and the problem was to maximize or minimize $\lambda_{1}$. The critical assumption on the domain $D$ in [13] is an "interior symmetry property," and with this assumption the authors further proved that, for the special case of two balls, $\lambda_{1}$ decreases when the center of the small ball (the obstacle) moves away from the center of the large ball, using a technique of domain reflection. For a wider 
class of domains containing obstacles, it was shown in [13] that the maximizing position of the obstacle resides in a special subset of $D$, which in the case where $D$ is convex corresponds to what has later come to be called the heart of $D$ in $[2,3] \nabla(D)$ (see the definition below). El Soufi and Kiwan [5, 6] have moreover proved other extensions of Hersch's result including one valid for the second eigenvalue $\lambda_{2}$.

The main aim of this paper is to establish a Hersch-type extremal result for the heat trace and the determinant of the Laplacian, as well as suitable generalizations for more general outer domains. We begin by stating the special case of domains bounded by balls: Given two positive numbers $R>r$ and a point $\mathbf{x} \in \mathbb{R}^{n},|\mathbf{x}|<R-r$, we denote by $\Omega(\mathbf{x})$ the domain of $\mathbb{R}^{n}$ obtained by removing the ball of radius $r$ centered at $\mathbf{x}$ from within the ball of radius $R$ centered at the origin.

Theorem 1.1. (i) For every $t>0$, the heat trace $Z_{\Omega(\mathbf{x})}(t)$ increases as the point $\mathbf{x}$ moves from the origin towards the boundary of the larger ball. In particular, $Z_{\Omega(\mathbf{x})}(t)$ is minimal when the balls are concentric $(\mathrm{x}=O)$ and maximal when the small ball touches the boundary of the largest ball $(|\mathbf{x}|=R-r)$.

(ii) The determinant of the Laplacian $\operatorname{det}(\Omega(\mathbf{x}))$ decreases as the point $\mathbf{x}$ moves from the origin towards the boundary of the largest ball. In particular, $\operatorname{det}(\Omega(\mathbf{x}))$ is maximal when the balls are concentric and minimal when the small ball touches the boundary of the largest ball.

Since $e^{-\lambda_{1}(\Omega) t}$ is the leading term in $Z_{\Omega}(t)$ as $t$ goes to infinity, it is clear that Theorem 1.1 implies the optimization result above for $\lambda_{1}$.

In order to state the more general theorem of which these propositions are special cases, we recall some definitions.

Definition 1.2. In [13], the domain $D$ was said to have the interior reflection property with respect to a hyperplane $P$ if there is a connected component $D_{s}$ of $D \backslash P$ whose reflection through $P$ is a proper subset of $D$. Any such $P$ will be called a hyperplane of interior reflection for $D$. The component $D_{s}$ will be called the small side of $D$ (with respect to $P$ ) and the other connected component $D_{b}$ will be called the big side.

The heart of $D$ is defined as the set of points $\mathbf{x} \in D$ so that there is no hyperplane of interior reflection passing through $\mathbf{x}$. We denote it $\nabla(D)$.

In the case where $D$ is convex, the heart $\varnothing(D)$ can be viewed as the closure in $D$ of the intersection of all the big sides with respect to the hyperplanes of interior reflection of $D$. This definition is equivalent to that introduced in $[2,3]$, where several properties of the heart of a convex domain are investigated. 
The heart of a bounded domain $D$ is a nonempty closed subset of $D$. Moreover, for a strictly convex bounded $D$ one has $\operatorname{dist}(\nabla(D), \partial D)>0$. We observe that for the ball and for many other domains with sufficient symmetry to identify an unambiguous center, the heart is simply the center.

Theorem 1.3. Let $D$ be a bounded $C^{2}$ domain of $\mathbb{R}^{n}$ and let $r>0$. For every $\mathbf{x} \in D$ so that the ball $B(\mathbf{x}, r)$ is contained in $D$, we set $\Omega(\mathbf{x})=D \backslash B(\mathbf{x}, r)$.

(i) For every $t>0$, let $\mathbf{x}_{0}(t)$ and $\mathbf{x}_{1}(t)$ be such that the function $\mathbf{x} \mapsto Z_{\Omega(\mathbf{x})}(t)$ achieves its minimum at $\mathbf{x}_{0}(t)$ and its maximum at $\mathbf{x}_{1}(t)$. Then $\mathbf{x}_{0}(t)$ belongs to $\varnothing_{r}(D):=\varnothing(D) \cap\{\mathbf{x}: \operatorname{dist}(\mathbf{x}, \partial D) \geq r\}$ and $\mathbf{x}_{1}(t)$ is either an interior point of $\nabla(D)$ or $\operatorname{dist}\left(\mathbf{x}_{1}(t), \partial D\right)=r$ (i.e. $B\left(\mathbf{x}_{\mathbf{1}}(\mathbf{t}), r\right)$ touches the boundary of $\left.D\right)$.

(ii) Let $\mathbf{x}_{0}^{\prime}$ and $\mathbf{x}_{1}^{\prime}$ be such that the regularized determinant of the Laplacian $\mathbf{x} \mapsto \operatorname{det}(\Omega(\mathbf{x}))$ achieves its maximum at $\mathbf{x}_{0}^{\prime}$ and its minimum at $\mathbf{x}_{1}^{\prime}$. Then $\mathbf{x}_{0}^{\prime}$ belongs to $\nabla_{r}(D)$ and $\mathbf{x}_{1}^{\prime}$ is either an interior point of $\odot(D)$ or $\operatorname{dist}\left(\mathbf{x}_{1}^{\prime}, \partial D\right)=r$.

We remark that these results imply those of [13], by a straightforward consideration of the limit $t \rightarrow \infty$.

We conjecture that at least for a convex domain $D$, the maximum of $Z_{\Omega(\mathbf{x})}(t)$ (resp. the minimum of $\operatorname{det}(\Omega(\mathbf{x}))$ ) is achieved when $B(\mathbf{x}, r)$ touches the boundary of $D$. This is the case for example when a convex domain $D$ admits a hyperplane of symmetry since then, $\operatorname{int}(\nabla(D))=\emptyset$. On the other hand, we believe that $\mathbf{x}_{0}$ should be independent of $t$ at least under some convexity conditions.

Remark 1. We shall approach the analysis of the regularized determinant via the spectral zeta function $\zeta(s)$, which for large $s$ is related to $Z(t)$ by an order-preserving integral transform. As in [12], transform theory can similarly be used to obtain corollaries for many further functions, e.g., Riesz means, with respect to the optimal position of an obstacle.

\section{Proof of ReSUlts}

Let $\Omega \subset \mathbb{R}^{n}$ be a domain of the form $\Omega=D \backslash B$, where $D$ is a simply connected bounded domain and $B$ is a convex domain such that the closure of $B$ is contained in $D$.

Assume that the domain $D$ has the interior reflection property with respect to a hyperplane $P$ about which the set $B$ is reflection-symmetric. 
Our strategy is to consider a displacement of the obstacle by $\varepsilon$ in a certain direction and to show that $Z_{\varepsilon}(t)$ is monotonically increasing in that direction.

Thus let $V$ be the unit vector perpendicular to $P$ and pointing in the direction of the small side $D_{s}$. For small $\varepsilon>0$, we translate $B$ by a distance $\varepsilon$ in the direction of $V$ and set $B_{\varepsilon}:=B+\varepsilon V$ and $\Omega_{\varepsilon}:=D \backslash B_{\varepsilon}$. The results of this paper rely on the following Proposition.

Proposition 1. Assume that the domain $D$ has the interior reflection property with respect to a hyperplane $P$ about which the set $B$ is reflection-symmetric and consider displacements as described above. Then, except possibly for a finite set of values of $t>0$,

$$
\left.\frac{\partial}{\partial \varepsilon} Z_{\Omega_{\varepsilon}}(t)\right|_{\varepsilon=0}>0
$$

and

$$
\left.\frac{d}{d \varepsilon} \operatorname{det}\left(\Omega_{\varepsilon}\right)\right|_{\varepsilon=0}<0 .
$$

Proof. The heat kernel $K$ on $\Omega$ under the Dirichlet boundary condition is defined as the fundamental solution of the heat equation, that is

$$
\left\{\begin{array}{l}
\left(\frac{\partial}{\partial t}-\Delta_{y}\right) K(t, \mathbf{x}, \mathbf{y})=0 \text { in } \Omega \\
K\left(0^{+}, \mathbf{x}, \mathbf{y}\right)=\delta_{\mathbf{x}}(\mathbf{y}) \\
K(t, \mathbf{x}, \mathbf{y})=0 \quad \forall \mathbf{y} \in \partial \Omega
\end{array}\right.
$$

The relationship between the heat kernel and the spectral decomposition of the Dirichlet Laplacian in $\Omega$ is given by

$$
K(t, \mathbf{x}, \mathbf{y})=\sum_{k \geq 1} e^{-\lambda_{k}(\Omega) t} u_{k}(\mathbf{x}) u_{k}(\mathbf{y}),
$$

where $\left(u_{k}\right)_{k \geq 1}$ is an $L^{2}(\Omega)$-orthonormal family of eigenfunctions satisfying

$$
\left\{\begin{array}{l}
-\Delta u_{k}=\lambda_{k}(\Omega) u_{k} \text { in } \Omega \\
u_{k}=0 \text { on } \partial \Omega .
\end{array}\right.
$$

The heat trace is then given by

$$
Z_{\Omega}(t)=\int_{\Omega} K(t, \mathbf{x}, \mathbf{x}) d x=\sum_{k \geq 1} e^{-\lambda_{k}(\Omega) t} .
$$

We recall the following Hadamard-type formula for $\varepsilon \mapsto Z_{\Omega_{\varepsilon}}(t)$ : 


$$
\left.\frac{\partial}{\partial \varepsilon} Z_{\Omega_{\varepsilon}}(t)\right|_{\varepsilon=0}=-\frac{t}{2} \int_{\partial \Omega} v \Delta K(t, \mathbf{x}, \mathbf{x}) d x
$$

where $v=X \cdot \nu$ is the component of the deformation vectorfield $X$ in the direction of the inward unit normal $\nu$ and $\Delta K(t, \mathbf{x}, \mathbf{x})$ stands for the Laplacian of the function $\mathbf{x} \mapsto K(t, \mathbf{x}, \mathbf{x})$ (cf. [4, Theorem 4.1]. For more information about Hadamard deformations we refer to $[9,10,15]$.) Since the outer boundary $\partial D$ of $\Omega_{\varepsilon}$ is fixed and the inner boundary $\partial B_{\varepsilon}$ is translated in the direction of $V$, the function $v$ vanishes on $\partial D$ and coincides with $V \cdot \nu$ on $\partial B$. Thus,

$$
\left.\frac{\partial}{\partial \varepsilon} Z_{\Omega_{\varepsilon}}(t)\right|_{\varepsilon=0}=-\frac{t}{2} \int_{\partial B} \Delta K(t, \mathbf{x}, \mathbf{x}) V \cdot \nu(\mathbf{x}) d x .
$$

Let $B_{s}$ be the half of $B$ contained in the small side $D_{s}$ of $D$ and $(\partial B)_{s}=\partial B \cap D_{s}$. Using the symmetry assumption on $B$ with respect to $P$ we obtain

$$
\left.\frac{\partial}{\partial \varepsilon} Z_{\Omega_{\varepsilon}}(t)\right|_{\varepsilon=0}=-\frac{t}{2} \int_{(\partial B)_{s}}\left(\Delta K(t, \mathbf{x}, \mathbf{x})-\Delta K\left(t, \mathbf{x}^{*}, \mathbf{x}^{*}\right)\right) V \cdot \nu(\mathbf{x}) d x
$$

where $\mathbf{x}^{*}$ stands for the reflection of $\mathbf{x}$ through $P$.

Define the function $\phi(t, \mathbf{x}, \mathbf{y})=K(t, \mathbf{x}, \mathbf{y})-K\left(t, \mathbf{x}^{*}, \mathbf{y}^{*}\right)$ on $(0, \infty) \times$ $\Omega_{s} \times \Omega_{s}$ with $\Omega_{s}=D_{s} \backslash B_{s}$.

Claim : For all $(t, \mathbf{x}, \mathbf{y}) \in(0, \infty) \times \Omega_{s} \times \Omega_{s}, \phi(t, \mathbf{x}, \mathbf{y}) \leq 0$.

Indeed, for all $\mathbf{x} \in \bar{\Omega}_{s}$, the function $(t, \mathbf{y}) \mapsto \phi(t, \mathbf{x}, \mathbf{y})$ is a solution of the following parabolic problem :

$$
(*)\left\{\begin{array}{l}
\left(\frac{\partial}{\partial t}-\Delta_{y}\right) \phi(t, \mathbf{x}, \mathbf{y})=0 \text { in } \Omega_{s} \\
\phi\left(0^{+}, \mathbf{x}, \mathbf{y}\right)=0 .
\end{array}\right.
$$

Let us check the sign of $\phi(t, \mathbf{x}, \mathbf{y})$ on $(0, \infty) \times \partial \Omega_{s} \times \partial \Omega_{s}$. Notice that $\partial \Omega_{s}$ is the union of three components : $(\partial D)_{s},(\partial B)_{s}$ and $\Omega \cap P$. First, from the boundary conditions, if $\mathbf{x} \in(\partial B)_{s}$ or $\mathbf{y} \in(\partial B)_{s}$, then $\phi(t, \mathbf{x}, \mathbf{y})=0$. On the other hand, $K(t, \mathbf{x}, \mathbf{y})$ vanishes as soon as $\mathbf{x} \in$ $(\partial D)_{s}$ or $\mathbf{y} \in(\partial D)_{s}$, which implies $\phi(t, \mathbf{x}, \mathbf{y})=-K\left(t, \mathbf{x}^{*}, \mathbf{y}^{*}\right) \leq 0$. It remains to consider the case where both $\mathbf{x}$ and $\mathbf{y}$ belong to $\Omega \cap P$. In this case we have $\mathbf{x}^{*}=\mathbf{x}, \mathbf{y}^{*}=\mathbf{y}$ and $\phi(t, \mathbf{x}, \mathbf{y})=0$.

In conclusion, for any $\mathbf{x}$ on $\partial \Omega_{s}$, the function $(t, \mathbf{y}) \mapsto \phi(t, \mathbf{x}, \mathbf{y})$ is everywhere nonpositive on the boundary of the cylinder $(0, \infty) \times \Omega_{s}$ which implies, thanks to $(*)$ and the parabolic maximum principle (see e.g., [7]), that $\phi(t, \mathbf{x}, \mathbf{y}) \leq 0$ for all $t>0$ and all $\mathbf{y} \in \bar{\Omega}_{s}$.

Now, from the symmetry of $\phi$ with respect to $\mathbf{x}$ and $\mathbf{y}$, the function $(t, \mathbf{x}) \mapsto \phi(t, \mathbf{x}, \mathbf{y})$ satisfies the same parabolic system as $(*)$. Since we 
now know that $\forall \mathbf{y} \in \bar{\Omega}_{s}$, the function $(t, \mathbf{x}) \mapsto \phi(t, \mathbf{x}, \mathbf{y})$ is everywhere nonpositive on the boundary of the cylinder $(0, \infty) \times \Omega_{s}$, the parabolic maximum principle then implies the non positivity of $\phi(t, \mathbf{x}, \mathbf{y})$ in the whole cylinder $(0, \infty) \times \Omega_{s} \times \Omega_{s}$.

Claim : $\Delta \phi(t, \mathbf{x}, \mathbf{x}) \leq 0$ for all $(t, \mathbf{x}) \in(0, \infty) \times(\partial B)_{s}$.

As we have seen, the function $\mathbf{x} \in \Omega_{s} \mapsto \phi(t, \mathbf{x}, \mathbf{x})$ achieves its maximum at the boundary. Moreover, since $K(t, \mathbf{x}, \mathbf{x})=\sum_{k \geq 1} e^{-\lambda_{k}(\Omega) t} u_{k}(\mathbf{x})^{2}$, the function $\phi(t, \mathbf{x}, \mathbf{x})$ vanishes quadratically on $(\partial B)_{s}$. Thus, for any $\mathbf{x}_{0} \in(\partial B)_{s}$

$$
\nabla \phi\left(t, \mathbf{x}_{0}, \mathbf{x}_{0}\right)=0
$$

Taking polar coordinates $(\rho, \sigma)$ centered at the center of $B$ and writing $\mathbf{x}_{0}=\left(\rho_{0}, \sigma_{0}\right)$ we see that, since all the first derivatives of $\mathbf{x} \in \Omega_{s} \mapsto$ $\phi(t, \mathbf{x}, \mathbf{x})$ vanish at $\mathbf{x}_{0}$,

$$
\Delta \phi\left(t, \mathbf{x}_{0}, \mathbf{x}_{0}\right)=\frac{\partial^{2}}{\partial \rho^{2}} \phi\left(t, \rho_{0}, \sigma_{0}, \rho_{0}, \sigma_{0}\right) .
$$

This is nonpositive, since $\rho \mapsto \phi\left(t, \rho, \sigma_{0}, \rho, \sigma_{0}\right)$ achieves its maximum at $\rho=\rho_{0}$.

Claim : Except possibly for a finite set of values of $t>0$,

$$
\left.\frac{\partial}{\partial \varepsilon} Z_{\Omega_{\varepsilon}}(t)\right|_{\varepsilon=0}>0
$$

It is clear that the product $V . \nu(\mathbf{x})$ is positive at every point $\mathbf{x}$ of the hemisphere $(\partial B)_{s}$. From equation (5) and the previous claim we then deduce that, $\forall t>0$,

$$
\left.\frac{\partial}{\partial \varepsilon} Z_{\Omega_{\varepsilon}}(t)\right|_{\varepsilon=0} \geq 0
$$

To show that this quantity cannot vanish at more than a finite set of values of $t$, we shall show that it is analytic as a function of $t$ in the open right half plane, and strictly positive for real values of $t$ sufficiently large. By the unique continuation theorem an analytic function that vanishes on a set with a point of accumulation is identically zero, which would pose a contradiction.

To establish analyticity of (1), we argue as follows. Because each eigenvalue of the Laplacian is at most finitely degenerate, according to [17] there is a numbering of the eigenvalues $\left\{\lambda_{k}(\epsilon)\right\} \rightarrow\left\{\Lambda_{k}(\epsilon)\right\}$ for which each $\Lambda_{k}(\epsilon)$ is analytic in $\epsilon$ in a neighborhood of $\epsilon=0$. (Using this numbering, which is important only in a neighborhood of a degenerate eigenvalue, does not alter $Z(t)$ as defined in (1).) In consequence of the Hadamard formula for the derivative of an eigenvalue, $\left.\frac{\partial \Lambda_{k}}{\partial \epsilon}\right|_{\varepsilon=0}$ is dominated in norm by the integral of the square of the normal derivative of an associated $L^{2}$ normalized eigenfunction $u_{k}$ over the boundary of 
the obstacle. For $C^{2}$ domains, both $\left\|u_{k}\right\|_{\infty}$ and $\left\|\nabla u_{k}\right\|_{\infty}$ are bounded by constants times finite powers of $\lambda_{k}[11,14]$, which in turn $\sim k^{\frac{2}{n}}$ by the Weyl law. It follows that both the series (4) and its term-by-term derivative with respect to $\epsilon$ converge uniformly on each set of the form $\{$ Re $t \geq C>0\}$.

To finish the argument, we observe that by differentiating (1),

$$
\left.\frac{\partial}{\partial \varepsilon} Z_{\Omega_{\varepsilon}}(t)\right|_{\varepsilon=0}=e^{-\lambda_{1} t}\left(-\left.\frac{\partial \lambda_{1}}{\partial \varepsilon}\right|_{\varepsilon=0}+0\left(e^{-\left(\lambda_{2}-\lambda_{1}\right) t}\right)\right) .
$$

this is strictly positive for large $t$ because $\lambda_{1}$ is nondegenerate and $\left.\frac{\partial \lambda_{1}}{\partial \varepsilon}\right|_{\varepsilon=0}<0$ by [13].

This completes the proof of (2). The proof of (3) relies on the following formula which is valid for every complex number $s$ with $\operatorname{Re} s>\frac{n}{2}$

$$
\zeta_{\varepsilon}(s):=\sum_{k \geq 1} \frac{1}{\lambda_{k}^{s}\left(\Omega_{\varepsilon}\right)}=\frac{1}{\Gamma(s)} \int_{0}^{\infty} Z_{\Omega_{\varepsilon}}(t) t^{s-1} d t .
$$

It is well known that the function $Z_{\Omega_{\varepsilon}}$ satisfies

$$
Z_{\Omega_{\varepsilon}}(t) \sim \sum_{k \geq 0} a_{k} t^{\frac{(k-n)}{2}} \quad \text { as } t \rightarrow 0,
$$

where $a_{k}$ is a sequence of real numbers that only depend on the geometry of the boundary of $\Omega_{\varepsilon}$ (see e.g. [1]). Hence, it is clear that the coefficients $a_{k}$ in (6) are independent of $\varepsilon$.

We set

$$
\tilde{Z}_{\Omega_{\varepsilon}}(t)=Z_{\Omega_{\varepsilon}}(t)-\sum_{k=0}^{n} a_{k} t^{\frac{(k-n)}{2}},
$$

so that $\tilde{Z}_{\Omega_{\varepsilon}}(t) / \sqrt{t}$ is a bounded function in a neighborhood of $t=0$. We also introduce the meromorphic function

$$
R(s)=\frac{1}{\Gamma(s)} \sum_{k=0}^{n} a_{k} \int_{0}^{1} t^{s-1+(k-n) / 2} d t=\frac{1}{\Gamma(s)} \sum_{k=0}^{n} \frac{a_{k}}{s-(n-k) / 2},
$$

which has poles at $1 / 2,1,3 / 2,2, \cdots, n / 2$. (Note that $s=0$ is not a pole since $\frac{1}{s \Gamma(s)}$ a holomorphic function on $\mathbb{C}$.)

Consequently,

$$
\zeta_{\varepsilon}(s)=\frac{1}{\Gamma(s)} \int_{0}^{1} Z_{\Omega_{\varepsilon}}(t) t^{s-1} d t+\frac{1}{\Gamma(s)} \int_{1}^{\infty} Z_{\Omega_{\varepsilon}}(t) t^{s-1} d t
$$


where the last term is an entire function of $s$, since $Z_{\Omega_{\varepsilon}}(t)$ behaves as $e^{-\lambda_{1}\left(\Omega_{\varepsilon}\right)}$ when $t \rightarrow+\infty$. Thus,

$$
\zeta_{\varepsilon}(s)=R(s)+\frac{1}{\Gamma(s)} \int_{0}^{1} \tilde{Z}_{\Omega_{\varepsilon}}(t) t^{s-1} d t+\frac{1}{\Gamma(s)} \int_{1}^{\infty} Z_{\Omega_{\varepsilon}}(t) t^{s-1} d t,
$$

where the equality holds, by unique continuation, in the neighborhood of $s=0$. The reciprocal gamma function $f(s):=\frac{1}{\Gamma(s)}$ vanishes at $s=0$ and satisfies $f^{\prime}(0)=1$. Therefore,

$$
\zeta_{\varepsilon}^{\prime}(0)=R^{\prime}(0)+\int_{0}^{1} \tilde{Z}_{\Omega_{\varepsilon}}(t) t^{-1} d t+\int_{1}^{\infty} Z_{\Omega_{\varepsilon}}(t) t^{-1} d t .
$$

Since $\operatorname{det}\left(\Omega_{\varepsilon}\right)=e^{-\zeta_{\varepsilon}^{\prime}(0)}$, we derive (3) from (7) and (2).

Proof of Theorem1.3. Let $D$ be a domain of $\mathbb{R}^{n}$ and let $r>0$ be less than the inradius of $D$. Let $\mathbf{x}$ be a point of $\{\mathbf{x}: \operatorname{dist}(\mathbf{x}, \partial D)>r\} \subset$ $D$. If $\mathbf{x} \notin \mathcal{Q}(D)$, by definition there exists a hyperplane of interior reflection $P$ of $D$ passing through $\mathbf{x}$. Applying Proposition 1, we see that the function is monotonically nonincreasing as the obstacle moves towards $\varnothing(D)$. The minimum is therefore achieved in $\varnothing(D)$. At this stage we have not eliminated the possibility of a value of $t=t_{0}$ and a line segment exiting from $\nabla(D)$ along which $Z_{\Omega(\mathbf{x})}\left(t_{0}\right)$ is constant and equal to its minimum with respect to $\mathbf{x}$. This possibility is, however, excluded by the analyticity of $Z_{\Omega(\mathbf{x})}(t)$ and the fact that for values of $t$ arbitrarily close to $t_{0}, Z_{\Omega(\mathbf{x})}(t)$ is strictly monotonic on the segment. As $\nabla(D)$ is closed in $D$ the minimizing point $\mathbf{x}_{0}(t)$ can never belong to the complement of $\varnothing(D)$.

The same arguments show that if $\mathbf{x} \mapsto Z_{\Omega(\mathbf{x})}(t)$ achieves its maximum at a point $\mathbf{x}_{1}(t)$, then either $\operatorname{dist}\left(\mathbf{x}_{1}(t), \partial D\right)=r$ or $\mathbf{x}_{1}(t)$ belongs to the interior of $\varnothing(D)$.

Acknowledgments The authors gratefully note that much of this work was done at the Centro de Giorgi in Pisa and while the second author was a visiting professor at the Université François Rabelais, Tours, France.

\section{REFERENCES}

[1] Thomas P. Branson and Peter B. Gilkey. The asymptotics of the Laplacian on a manifold with boundary. Comm. Partial Differential Equations, 15(2):245272, 1990.

[2] Lorenzo Brasco and Rolando Magnanini. The heart of a convex body. In Geometric properties for parabolic and elliptic PDE's, volume 2 of Springer INdAM Ser., pages 49-66. Springer, Milan, 2013. 
[3] Lorenzo Brasco, Rolando Magnanini, and Paolo Salani. The location of the hot spot in a grounded convex conductor. Indiana Univ. Math. J., 60(2):633-659, 2011.

[4] Ahmad El Soufi and Saïd Ilias. Domain deformations and eigenvalues of the Dirichlet Laplacian in a Riemannian manifold. Illinois J. Math., 51(2):645-666 (electronic), 2007.

[5] Ahmad El Soufi and Rola Kiwan. Extremal first Dirichlet eigenvalue of doubly connected plane domains and dihedral symmetry. SIAM J. Math. Anal., 39(4):1112-1119, 2007/08.

[6] Ahmad El Soufi and Rola Kiwan. Where to place a spherical obstacle so as to maximize the second Dirichlet eigenvalue. Commun. Pure Appl. Anal., 7(5):1193-1201, 2008.

[7] Lawrence C. Evans. Partial differential equations, volume 19 of Graduate Studies in Mathematics. American Mathematical Society, Providence, RI, second edition, 2010.

[8] G. Faber. Beweis, dass unter allen homogenen membranen von gleicher fläche und gleicher spannung die kreisförmige den tiefsten grundton gibt. Sitz. Ber. Bayer. Akad. Wiss., pages 169-172, 1923.

[9] P. R. Garabedian. Partial differential equations. AMS Chelsea Publishing, Providence, RI, 1998. Reprint of the 1964 original.

[10] P. R. Garabedian and M. Schiffer. Convexity of domain functionals. J. Analyse Math., 2:281-368, 1953.

[11] D. Grieser. Uniform bounds for eigenfunctions of the Laplacian on manifolds with boundary. Comm. Partial Differential Equations, 27(7-8):1283-1299, 2002.

[12] Evans M. Harrell, II and Lotfi Hermi. On Riesz means of eigenvalues. Comm. Partial Differential Equations, 36(9):1521-1543, 2011.

[13] Evans M. Harrell, II, Pawel Kröger, and Kazuhiro Kurata. On the placement of an obstacle or a well so as to optimize the fundamental eigenvalue. SIAM J. Math. Anal., 33(1):240-259 (electronic), 2001.

[14] Andrew Hassell and Terence Tao. Upper and lower bounds for normal derivatives of Dirichlet eigenfunctions. Math. Res. Lett., 9(2-3):289-305, 2002.

[15] Daniel Henry. Geometric theory of semilinear parabolic equations, volume 840 of Lecture Notes in Mathematics. Springer-Verlag, Berlin, 1981.

[16] Joseph Hersch. The method of interior parallels applied to polygonal or multiply connected membranes. Pacific J. Math., 13:1229-1238, 1963.

[17] Tosio Kato. Perturbation theory for linear operators. Classics in Mathematics. Springer-Verlag, Berlin, 1995. Reprint of the 1980 edition.

[18] S. Kesavan. On two functionals connected to the Laplacian in a class of doubly connected domains. Proc. Roy. Soc. Edinburgh Sect. A, 133(3):617-624, 2003.

[19] E. Krahn. Über eine von Rayleigh formulierte Minimaleigenschaft des Kreises. Math. Ann., 94(1):97-100, 1925.

[20] Richard Snyder Laugesen and Carlo Morpurgo. Extremals for eigenvalues of Laplacians under conformal mapping. J. Funct. Anal., 155(1):64-108, 1998.

[21] J. M. Luttinger. Generalized isoperimetric inequalities. J. Mathematical Phys., 14:586-593, 1973.

[22] B. Osgood, R. Phillips, and P. Sarnak. Extremals of determinants of Laplacians. J. Funct. Anal., 80(1):148-211, 1988. 
[23] D. B. Ray and I. M. Singer. R-torsion and the Laplacian on Riemannian manifolds. Advances in Math., 7:145-210, 1971.

[24] Ken Richardson. Critical points of the determinant of the Laplace operator. $J$. Funct. Anal., 122(1):52-83, 1994.

E-mail address: elsoufi@univ-tours.fr

E-mail address: harrell@math.gatech.edu 